\title{
A KINETIC INTERPRETATION OF OSMOTIC PRESSURE
}

BY M. M. GARVER

In the modern theory of dilute solutions, what is known as osmotic pressure plays a very prominent part, a fundamental assumption with some writers being that the observed pressure is due to an "expansive force" of the dissolved substance exerting a pressure analogous to that of a gas, or, as other writers put it, "a dissolved substance in solution 'obeys' the gas laws." Notwithstanding the very great success of the theory in explaining phenomena and in furnishing a basis for deducing numerical relations by assuming that the numerical values of the general gas equation apply to the dissolved substance in dilute solutions, some writers are careful to state that their deductions are entirely independent of any hypothesis as to the cause of the observed pressure. This indicates that the gas analogy hypothesis does not satisfy their minds--that there is a lack of physical verisimilitude in the hypothesis, although the numerical relations based on experience are accepted as being beyond dispute. If the function of a scientific explanation of a physical phenomenon is to exhibit to the mind the mechanical relations of the various elements of the phenomenon, then it must be admitted that there is a gap in the current explanation of "osmotic pressure" that many minds are unable to bridge. This sometimes leads to actually grotesque or absurd statements in some of the text-books. A satisfactory mechanical explanation of the osmotic process entirely free from arbitrarily assumed "forces" and "tendencies," and that is at the same time consistent with all the known facts, has apparently not yet been developed. Scientifically considered, the interpretation of a phenomenon is often quite as important as the establishment of the existence of the phenomenon itself.

If we examine the two leading hypotheses at present 
current we shall find that besides being philosophically weak, they fail entirely to explain, and are inconsistent with, certain other well-established experimental facts. By the "two leading hypotheses" I mean, first, that the external work done by the osmotic cell, or other device using a semi-permeable wall, is due to an expansive force exerted by the dissolved substance 'tending' to occupy a greater volume, and secondly, the alternative hypothesis that the increase in volume and consequent increase in pressure observed in osmotic experiments are due to some kind of attractive force exerted between the molecules of solute and solvent. Both hypotheses can be shown to be untenable. If then, all attempts at explanation based on these two hypotheses are definitely and distinctly eliminated as being untenable, we are free to re-examine the subject with a riew to finding some hypothesis that is tenable.

We know from themodynamics that the extemal work which may be done by a substance in passing from the state A to the state $B$, is a maximum when the process is reversible and is the same for all reversible processes between the same two states, the result depending only on the initial and final states. Therefore, if we dilute a solution isothermally and reversibly from the state $A$ to the state $B$, the external work done will depend only upon the initial and final states. For the details of such a process, using a scmipermeable piston and "explained" in terms of the "expansive force" of the dissolved substance, the reader is referred to the text-books. An equal amont of external work may, however, be done by an entirely different process, using a deferent deviece working isothernally and revereibly between any two chosent states of dilution, where neither an expansive force of the dissolved sthitance nor any specific attactive forces of ans kind between the solute and solvent other than these wheh come inte pay during ordinary condensation and vaporizat

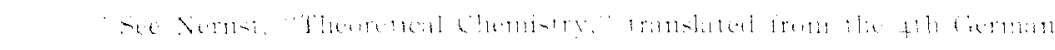

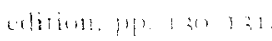


tion, can be admitted as taking any part whatever in the production of the external work.

The experimental fact that the saturated vapor of a dilute solution of a non-volatile solid in a liquid solvent exerts less pressure than the saturated vapor of the pure solvent at the same temperature may be utilized to carry out a reversible isothermal process between any two given states of dilution. By the thermodynamic principle just stated the amount of external work done must be the same in the two cases, since both are reversible; therefore, the ultimate source of the work done must be the same in both; at least, any hypothesis worth considering must include both. Philosophically it would be absurd to invent separate hypotheses for each separate casc.

If two vessels, both containing the same liquid, one of which in addition, contains a foreign solid substance in solution, be placed side by side in a space from which the air is exhausted, isothermal distillation will take place, the rolunie of the solution increasing at the expense of the volume of the pure solvent. Now suppose that instead of placing them side by side, that the solution of strength $A$ be placed on a shelf at a height $h_{1}$ centimeters above the level of the pure solvent. To insure equalization of temperatures. suppose both ressels to be put in thermal contact with the same good conductor of heat. Over all place a cover and exhaust the air. The height $h$ may be so chosen that the rapor of the solution A will be in linetic equilibrinn with the vapor of the pure solvent at that height. There will then be no change of volume of the solution. Now suppose that at the height h. the rapos of the solution of strength $B$ will be in equilibrium with the rapor of the pure solvent at that leciglet where $h_{g}<h_{\text {, and }} \mathrm{B}$ is more dilute than $A$. By sowly changing the elevation of the solution so slowly that its rapor mat be regarded as always in epuilibrium? with the -mrounding vapor of the pure solvent the degree of dilution may be increased or decrated at will between any chosen degrees of dilution Here we have at reverible inothermal proeses. the work done 
between the two limits $h_{1}$ and $h_{2}$ being evidently $g \int_{h_{1}}^{h_{2}} h d m$ where $d m$ represents the increment of the mass of solvent at the height $h$, and may be either positive or negative. If we express mass in terms of volume and density we will have

$$
\mathrm{W}=\rho g \int_{h_{1}}^{h_{\mathrm{a}}} h d v
$$

If $v$ be expressed as a function of $h$, the work done on or by the solution between the limits may be determined, but the important point to observe, is, that the work is independent of any force function whatever, except gravity, and that may be included as a constant.

By the first law of thermodynamics, heat must disappear when mechanical work is done. In the above ideal experiment, the heat equivalent of the external work done, it may be seen, will come from the cooling of the vapor of the pure solvent, if it expand isentropically, or from the environment, if the expansion be isothermal. In either case no work is done by the expanding solute any more than if additional liquid solvent were added directly by hand. In the osmotic cell the heat equivalent of the work done is assumed to come from the cooling of the expanding solute acting like a gas! But since the dissolved substance could not be cooled without cooling the surrounding solvent the solution, if the supposition be correct, must be cooled when mechanical work is done unless heat be supplied by the surroundings. By reference to the above experiment it is seen that the solution would be warmed by the condensing vapor while doing mechanical work, were it not in thermal communication with the pure solvent. However, the important point is, that since the same amount of work may be done in passing between two identical states by two different processes, in one of which it is evident that no expansive force, or attractive forces of any kind can have been the active cause, the hypothetical forces become unnecessary complications and explain nothing. 
But, moreover, other experiments show that the osmotic pressure is numerically equal to the pressure due to an equal number of gas molecules occupying the same volume at the same temperature as the solute. Hence if the increase of volume of solution be attributed to an attractive force of any kind acting between the molecules of solute and solvent, the hypothesis that the observed resistance to filtration is due to the bombarding molecules of the dissolved substance must be abandoned, since the resistance to filtration would necessarily be the sum of the two actions. Hence the simplest hypothesis is that the attractive forces are negligibly small. This conclusion accords with that previously drawn from the results of the isothermal distillation experiment.

It is just at this point that misinterpretation has crept in. The admission that the resistance to filtration is numerically equal to the pressure required to confine a gas having an equal number of molecules at the same temperature in the same volume as the dissolved substance does not by any means imply that the dissolved substance "tends to expand like a gas." This view is introduced surreptitiously, as it were, by the statement that the osmotic pressure "obeys" the gas laws. Numerical equality of certain relations does not warrant the use of the word "obey." Just here is the gap that requires bridging. No one who accepts Pascal's principle as a physical law can realize in thought just how, mechanically, an "expansive force," under the given conditions, can produce a seemingly negative pressure; hence the mind remains unsatisfied.

This mechanical difficulty may be overcome if one can mentally realize just what takes place during isothermal distillation. The vapor of the pure solvent has a greater density and consequently exerts a greater pressure at the same temperature than is exerted by the vapor of the same solvent when a foreign solid substance is held in solution. Hence when a solution and the pure solvent of the same solution are side by side at the same temperature, there results a transfer of material from the pure solvent to the solution with 
corresponding changes in volume. The mechanical paradox of the osmotic cell disappears entirely if we imagine the interstices of the cell-wall to be occupied only by the vapor of the solvent. Isothermal distillation then takes place through the cell-wall with a corresponding increase in the volume of the solution, until balanced by a pressure that forces the pure solvent out of the solution into the interstices of the cell-wall, $i . e$, until the filtration pressure is reached. Under these latter circumstances, as much solvent will leave as enters the cell. If this be admitted, merely as a provisional hypothesis, all paradoxes and mechanical difficulties disappear. It would be a simple explanation if true.

Now if we can explaitı isothermal distillation mechanically, and show that the result must be the same whether the interstices be regarded as filled with either the liquid or vapor, we shall have the clue to the mechanism of the osmotic cell.

It has been somewhere well stated that the study of osmotic phenomena has given a wonderful impulse to the kinetic theory. If this be so, it is highly probable that the phenomena of osmosis are explicable only in terms of kinetic phenomena. This latter statement seems to apply particularly to the doing of work by means of isothermal distillation, where no evidence of active forces is traceable. It seems but fitting, therefore, that we should seek to explain by mears of the kinetic theory the phenomena of the osmotic cell. Hence, with a view to bringing them immediately under the eye, the statement of a few of the deductions from the molecular kinetic theory, which if admitted will enable us to understand the mechanism of the osmotic cell, may here be permitted. Without going into any detail, a few of the generally accepted deductions which have a bearing on isothermal distillation and kindred phenomena may be stated.

1. The mean kinetic energy of translation of a molecule deperids upon its temperature and is the same for liquids and vapors at the same temperature.

2. The energy of translation of individual molecules in 
liquids and vapors varies between zero and some upper limit depending upon circumstances.

3. In the absence of chemical action, a gaseous mixture of different molecules of unequal masses, at the same temperature, tends toward a state having the same average energy of translation per molecule.

4. The vapor of a liquid is composed of those molecules, favorably situated, possessing sufficient energy of translation to enable them to escape through the non-homogeneous surface layer of the liquid

5. All cases of chemical equilibrium are kinetic, i. e., reaction balancing action.

Let us apply these deductions to dilute solutions. To fix the ideas let us take the case of a non-saline solution such as an aqueous solution of sugar. The molecule of sugar is ig times as heavy as the normal water molecule; hence will have in solution an average velocity lying between onefourth and one-fifth that of the normal vapor of water molecule. We shall find that, in general, the molecule of solid solute is heavier than that of the liquid solvent and will consequently possess a lower mean velocity of translation than the molecules of the solvent. Moreover, when a solid is dissolved in a liquid without producing chemical action or appreciable change of volume, there is a fall in temperature indicating that the solvent has given up energy to the solid in the process of changing the solid to the liquid state. It is evident that the average molecular energy of translation of the solvent has been lowered while that of the solid has been raised. The transferred energy could come only from those molecules of the solvent having more than the final average energy, so that the average of those having an excess of energy will be lowered more than the general azerage is lowered. If now, by the application of heat, the original temperature be restored, the original average molecular energy of translation will be restored, but not the original distribution of molecular energy. Since the mean of the energy of one-half of the molecules is lowered without dis- 
turbing the mean of the whole, the mean of the other half must be raised proportionately. The net effect is that the foreign molecule acts as though it were a carrier of energy from those molecules having an excess of energy to those having less than the average, with the result that, in a solution, the molecular energy is concentrated more closely about the mean molecular energy than is the case with the liquid pure solvent.

These considerations suggest a simple hypothesis which will enable us to understand and explain many of the phenomena connected with solutions. Let us suppose that in a solution the presence of a foreign non-volatile molecule has the effect of narrowing the range of the velocities of the lighter molecules of the solvent, so that while the average molecular energy of translation will depend upon the absolute temperature, there will be fewer molecules in a solution than in the normal pure solvent, possessing either exceptionally high or exceptionally low velocities.

It should be noticed particularly that the foreign substance is supposed to possess an average molecular energy of translation (not velocity) equal to that of the solvent; consequently the disturbing effect of the dissolved substance should be proportional to the number of molecules rather than to their mass, since the energy per molecule is independent of the mass.

This hypothesis, if accepted, will be found to enable us to explain many phenomena that are now obscure, such as:

The depressed vapor tension of solutions with accompanying elevation of boiling point and lowering of the freezing point.

Landsberger's method of determining boiling points of solutions.

Isothermal distillation.

The osmotic cell.

Hydro-diffusion.

These are all closely related phenomena and all should be explainable in terms of something they possess in common, i. e., by a single hypothesis. 
Let us apply the hypothesis first to the depressed vapor tension of solutions.

It appears from the kinetic theory of solutions ${ }^{1}$ that the non-homogeneous surface layer of a solution is free from the dissolved substance, while throughout the rest of the solution the dissolved substance is uniformly distributed. In that case, the absorbing power of the surface of the liquid solution for its vapor will be the same, at the same temperature, as a surface of the pure solvent, and, by Henry's law, will be proportional to the pressure of the vapor upon it. Since kinetic equilibrium ensues when as many molecules return to the liquid as leave it, the reduced vapor pressure of a solution indicates that fewer molecules will be returned to the liquid than in the case of the pure solvent. Therefore fewer molecules must have had sufficient energy to make their way out through the surface layer-a result evidently in accordance with the hypothesis.

The elevation of the boiling points and the depression of the freezing points of solutions may be taken as a measure of the degree of concentration of the molecular energies of the solvents about a mean energy, or, as we may put it, indicate the degree of narrowing of the range of molecular velocities. This may be best shown, perhaps, by first considering the relation of these points to the normal liquid solvent.

The boiling point of a normal pure solvent is that temperature at which the vapor in contact with the liquid exerts a pressure of one normal atmosphere. By the hypothesis, this temperature should be higher for a solution than for the pure solvent, for a higher temperature is required in order to give the necessary energy to a sufficient number of molecules to enable them to escape through the non-homogeneous surface layer, $i$. e., the boiling point of a solution should be raised.

As to the freezing point of the pure solvent, the liquid

${ }^{1}$ Boynton, "Kinetic Theory," p. $22 \mathrm{I}$. In the same work will be found references to the original papers warranting the above statements. 
must commence to solidify when the molecules of least energy take the solid form, keeping the temperature constant by giving out their heat of liquefaction until all are solidified.

$\mathrm{By}$ the hypothesis, in the case of a solution, the lower range of molecular energy is raised nearer the mean; consequently a lower temperature would be required before any of the molecules took the solid form, after which the process would proceed as with the pure solvent, i. e., the freezing points of solutions of solids should be lowered.

The fact that a solution may be boiled by passing into it the vapor of the pure solvent, although the boiling point of the solution may be considerably higher than the temperature of the entering vapor, is a standing paradox that I have never seen even an attempt to explain, although the experimental fact is utilized in Iandsberger's method of determining boiling points. If, as the hypothesis supposes, the foreign molecule absorbs energy readily from those molecules of the solvent having energy above the average, then the solution will absorb energy from the inflowing vapor until the solution gives off as much energy as it receives, $i$. $e$., boils, although the temperature of the solution is higher than the average molecular temperature of the inflowing vapor. In order that the temperature should continue to rise it is merely necessary that the heat given out by the absorted and condensed portion of the vapor should exceed the heat carried away by the unabsorbed and uncondensed portion of the infloring rapor.

The mechanism of isothermal distillation will be readily understood from the previous explanation of depressed vapor tension. The two surfaces. those of the pure solvent and of the solution, have sequal absorbing power at the same temperatures, but the solution furnishes less vapor than the pure solvent. Therefore the surace of the solution will absorb more that: it sipplies, or the volune of the solution mint in crease in the presenece of aturaterl vapor irom the pure solvent.

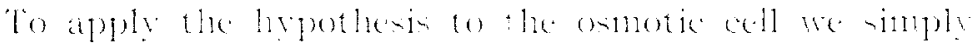


have to remember that although the average molecular energy of translation is the same for both the external pure solvent and the interior solution, a larger proportion of the molecules of the solvent than of the solution possesses exceptionally high velocities and energies. This fact is shown by the more copious vapor of the pure solvent. Hence if the interstices of the cell-wall are filled with vapor of the pure solvent having a pressure higher than the vapor pressure, due to the solution, condensation will take place where the vapor touches the solution, so that the volume of the solution will increase. This will continue so long as there is any break in the continuity of the liquid in the interstices. But suppose the external pure liquid solvent actually penetrates and touches the interior liquid solution. The only effect of this contact will be to remore the non-homogeneous surface laver, allowing even freer play to the molecules of the pure solvent. They can then pass directly into the solution at the point of contact without giving up a portion of their energy to the superficial layer. In the solution, they soon lose their exceptional energy, so that not as many molecules will be able to find their way out as in, unless aided by an interior hydrostatic pressure. Equilibrium, or the maximum pressure, will be reached when as nany molecules pass out as in, and this maximum pressure is eridently the pressure necessary to separate by filtration the solute fron the solvent.

The Mechanism of Diffurion..... If the effect of the presence of a dissolved instance in solution is to diminish the normal wide ratse of the molecular actirity of the solvent, it is at once apparent that diffusion of the dissolved substance throughout the liguid is quite a different proces from that ordinarily supposed. The discolved substance would " tend to become uniformly distributed," not on account of any inferent activity in the dicolved substance iteelf but be cause at crery point where there wat a diferenee in degree of dilution, the more atere molester of the solvent wonld make their way into the dethes olution faster than the molecules alreaty in the colution conth make their wat out This 
view, moreover, accords better with the generally accepted doctrine that molecular activity is the greater in the lighter molecule. If the energy of a molecule is independent of its mass, then molecules must have velocities inversely proportional to the square root of their masses. Since, in general, the dissolved solid will have a greater molecular weight than the liquid solvent, it is running counter to all accepted molecular theory to endow the heavy molecule in a mixture with special and unusual activity. It will be found that to transfer the activity from the solute to the liquid solvent will accord just as well with the experimental facts of diffusion. Take Fick's law, for instance. From the fact that the difference in vapor pressures is proportional to the difference in concentration, and the difference in vapor pressures represents the differences in activity of the solvent as modified by the foreign substance, the proposed hypothesis seems in no way in opposition to the observed facts. From the observed vapor pressures, it is evident that, in a solution possessing uniform temperature, no permanent state of equilibrium is possible except a uniform distribution of the dissolved substance.

State College, Pa., August, rgog 